interventions to address both patient and clinician barriers to video consultation uptake. Moreover, we understand that motivating and mobilising the care team was a key factor. Possible future work includes improving the sustainability of the interventions and assessing their efficacy in other care teams.

Improving quality and assessment of referrals to the Enfield Crisis Resolution and Home Treatment Team (ECRHTT)

Margarita Kousteni*, John Cousins, Ajay Mansingh, Maja Elia, Yumnah Ras, Mercedes Chavarri, Marilia Gougoulaki and Imo Akande

Barnet Enfield and Haringey Mental Health NHS Trust

${ }^{\star}$ Corresponding author.

doi: 10.1192/bjo.2021.541

Aims. Triaging referrals to crisis resolution and home treatment teams is a significant undertaking requiring experienced and dedicated staff. We observed that the volume of inappropriate referrals to ECRHTT was high, and that staff processing these often felt inexperienced or lacking in confidence to discharge them back to the referrers and signpost them to appropriate services.

The aims of this quality improvement project (QIP) were:

a) to reduce the number of inappropriate referrals received by the team

b) to reduce the number of inappropriate referrals accepted by the team

This would significantly improve access and flow to the service and facilitate better patient care.

Method. A pilot study was first completed of the quality (appropriateness/ inappropriateness) and source of all referrals to ECRHTT in January $2019(\mathrm{n}=177)$.

Subsequently, the consultant psychiatrist for ECRHTT based himself within the assessment team. He was able to closely monitor the referrals, at the same time as providing medical input to patients at their first point of contact. To evaluate the impact of this intervention, the percentage of inappropriate referrals accepted pre- and post-change was compared by re-auditing all referrals received in February $2019(\mathrm{n}=175)$.

Further interventions were instigated to improve referral quality. These included continuation of psychiatric medical input to the assessment team, teaching sessions for GPs and the crisis telephone service, and weekly meetings with psychiatric liaison and community mental health teams (CMHTs). Change was measured by reassessing the quality of all referrals made to ECRHTT in February $2020(\mathrm{n}=215)$.

Result. $46.9 \%$ of inappropriate referrals to ECRHTT were accepted in January 2019 compared to 16.9\% in February 2019 following the addition of medical input to the assessment team. The absolute difference was 30\% (95\% CI: $14 \%-44 \%, \mathrm{p}<0.001$ ).

$71 \%$ of referrals from GPs were inappropriate in January 2019 compared to $36 \%$ in February 2020 post-intervention (difference $35 \%$, 95\% CI: $8.84 \%-55.4 \%, \mathrm{p}<0.05)$. Inappropriate referrals from CMHTs decreased from $55.5 \%$ to $12 \%$ (difference $43.5 \%$, 95\% CI: $9.5 \%-70.3 \%, \mathrm{p}<0.05)$. Overall, the percentage of inappropriate referrals fell from $38 \%$ to $27.4 \%$, a difference of $10.6 \%$ (95\% CI: $1.3 \%-19.8 \%, \mathrm{p}<0.05$ ). The percentage of inappropriate referrals from liaison teams did not change significantly.
Conclusion. This piece of work shows that better engagement with referral sources significantly improved the quality of referrals made to ECRHTT. Interventions included medical input at the point of referral, teaching sessions for general practitioners as well as ongoing liaison with referring teams.

\section{Monitoring and investigation of tachycardia in patients receiving clozapine therapy; a quality improvement project}

Michael Kurkar*, Joshua Blair and Poppy Graham

Royal Oldham Hospital, Oldham, Pennine Care NHS Foundation Trust

${ }^{*}$ Corresponding author.

doi: 10.1192/bjo.2021.542

Aims. Clozapine is an antipsychotic agent with a number of significant physical health risks which necessitate monitoring, including cardiac complications such as myocarditis and cardiomyopathy. Reliable detection of cardiac complications requires active vigilance, with consideration for investigations if serious cardiac side effects are suspected (including electrocardiograms, echocardiograms and blood tests). There was dissatisfaction in the outpatient department about delays to taking action on abnormal physical observations, such as tachycardia. This raised safety concerns about how these delays would limit our ability to investigate and diagnose cardiac complications in a timely manner. We set a project aim to reduce the rate of retrospective action on abnormal physical observations, by half in the 4-month project timespan.

Method. All correspondence sent to the outpatient department from the local clozapine clinic was monitored and assessed for the need for further action or investigation, and the proportion of retrospective action needed was recorded. This was then monitored during implementation of project interventions, to detect any change in performance.

Result. Baseline monitoring showed retrospective action had to be taken on $41.2 \%$ of patients attending the clinic with abnormal physical observations, with significant delays up to 51 days later. Our initial intervention was the design of a clinical protocol to guide and signpost clinical staff at the time of the patient's attendance. Unfortunately, due to wider organisational barriers, this was not able to be implemented during the timescale of this project; however increased staff awareness during the protocol implementation process led to a reduction of retrospective action to $26.7 \%$. A follow-up intervention to increase staff awareness and education was carried out, with development of a poster for the clinical room. This approach maintained the improvement, with a further slight reduction to $26.3 \%$, representing a decrease of $37.5 \%$ from the baseline rate. A total of 106 patient letters were assessed during the project.

Conclusion. We believe that developing a clinical policy to use at the time of the patient's clinic attendance still remains the optimal intervention; a view backed up by this project's identified drivers for change. However, wider organisational barriers prevented the implementation of this policy, and overcoming these barriers are outside the scope and timescales of this project. This project demonstrated maintained, but sub-target, success with measures that increase staff education and awareness. However, it remains to be seen if this improvement will persist, and this would be a potential target for further QIP or monitoring. 
Child parent psychotherapy in the treatment of severe trauma in a 4-year-old child with co-occurring autism spectrum disorder

\section{Clare Lamb* and Barry O'Sullivan}

London Infant \& Family Team. South London \& Maudsley NHS Foundation Trust / NSPCC

${ }^{*}$ Corresponding author.

doi: $10.1192 /$ bjo.2021.543

Aims. This poster describes Child Parent Psychotherapy (CPP) in the treatment of severe trauma in a 4-year-old child with co-occurring Autism Spectrum Disorder (ASD).

Background. The London Infant and Family Team (LIFT) implements the New Orleans Intervention Model. It targets the mental health needs of under 5 year olds, providing evidence based assessments and interventions for infants, their parents and foster carers within the framework of the Family Court in England. The majority of children seen by LIFT have suffered severe trauma. LIFT delivers a range of interventions including CPP - a relational treatment for young children who have experienced trauma.

$\mathrm{CPP}$ seeks to intervene in a number of ways: provides developmental guidance, demonstrates that the child's behaviour has meaning and can be linked to past traumas, enables the child to have space to play and talk about what has happened, helps to name and contain emotions - supporting emotional regulation, and helps the dyad to understand each other. The dyadic relationship is key to the intervention - helping to establish safety for the child and strengthen the caregiver-child relationship, enabling the child to make sense of past experiences and learn new ways to express feelings. Exploration of trauma takes place through a combination of play and interpretations made by the clinician, who supports and holds in mind the experiences and history of both child and carer. There is evidence that CPP helps young traumatised children to become less anxious, more secure in their attachment relationships and more able to cue their needs. There is less evidence of the efficacy of CPP in the context of young children with a co-occurring diagnosis of ASD.

Method. The poster describes the assessment of a 4-year-old child of normal intelligence with a two year history of severe neglect, and physical and emotional abuse, who presented significant behavioural and emotional disturbance. Tools used to assess the child's behaviour, trauma symptoms and ASD are outlined. The process of CPP with the child and foster carer dyad is described. Outcome measures and symptom resolution are reported.

Conclusion. Co-occurrence of ASD did not prevent this child accessing trauma therapy. He engaged in symbolic play, made use of CPP interpretations, and achieved significant improvement in his symptoms. The differential diagnoses of trauma symptoms and ASD presenting in young children are discussed, alongside the importance of understanding and treating trauma in this context.

\section{Physical health assessment quality improvement project}

Maggie Lambert*

CAMHS, Northern Health Centre

${ }^{\star}$ Corresponding author.

doi: 10.1192/bjo.2021.544

Aims. My aim was to ensure at least $60 \%$ of clients in the Acute Day Unit have a 'physical screening tool' entry.
Background. As a GP starting training in psychiatry I am very aware of the importance of physical health and the overlap between physical health and mental health. It has been found that there is a 20 year mortality gap for men and 15 year mortality gap for women in people with mental health problems. Thorncroft described this as 'the scandal of premature mortality'.

Nice Guidelines state: 'Reducing premature mortality by improving physical healthcare for people with severe mental illness remains an NHS England priority. Funding has been made available to ensure that at least $60 \%$ of people who have severe mental illness receive NICE-recommended physical assessments and follow up from 2018/19 onwards.'

The Acute Day Unit seemed to be the ideal situation to try to address this problem as clients are with us for 6-8 weeks during which time their physical health as well as their mental health can be optimised.

Method. I emailed the whole team to invite ideas and questions regarding the QI project and discussed it further at the MDT meeting. It was important at the start to get the whole team on board. Having discussed it we decided to put six blocks of thirty minute slots weekly into the timetable for physical assessments. These were to be booked in by the client's care coordinator. I also added a column onto our team spreadsheet to input whether or not the physical assessment had been done. Frequent encouragements and reminders were sent round the team of which clients still needed a physical assessment.

Result. Before the changes were made $25 \%$ of clients were having their physical assessments done. After the changes were made $63 \%$ of clients had their physical assessment done, three of the twenty seven clients having only started at the day unit that week. Conclusion. Having made a change to the system of scheduling six regular slots for physical assessments there has been a dramatic rise in the number of clients having their physical assessment done. As this change has been to the system and will be continued automatically on the team calendar the improvement has been more easily sustained. We are keen to keep improving on this change with an ideal level of over $75 \%$ of clients having a physical health assessment.

\section{Staff's perspectives on physical activity in acute mental} health general adult wards

Nikoletta Lekka*, Samantha Nunns and Robert Verity

Sheffield Health and Social Care NHS Foundation Trust

${ }^{\star}$ Corresponding author.

doi: 10.1192/bjo.2021.545

Aims. Physical activity (PA) has multiple health benefits for people with severe mental illness (SMI). Nevertheless, people with SMI engage in less exercise and more sedentary behaviour than the general population. Additionally, inpatient settings can exacerbate barriers to PA and facilitate sedentary behaviour. Staffs attitudes towards PA promotion may influence patient engagement. The aim of this study was to explore staffs views on PA for acute psychiatric inpatients, including enablers and barriers.

Method. An online anonymous survey with free text was sent to all 85 multidisciplinary team (MDT) members of two acute general adult wards, including nurses, doctors and allied health professionals. A qualitative approach was used to gain deeper understanding of the participants' perspectives. Manual thematic analysis was completed to identify discrete themes.

Result. Response rate was $64 \%$, with 54 professionals responding. Notably, $100 \%$ agreed or strongly agreed that exercise is beneficial to physical and mental health. Nevertheless, $72 \%$ felt it was not 